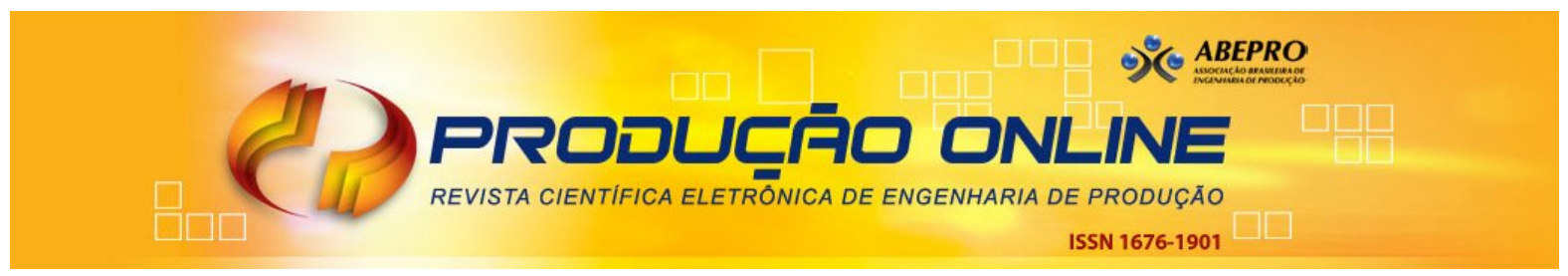

\title{
ESTRATÉGIA DE MANUTENÇÃO PREDITIVA NO DEPARTAMENTO GRÁFICO DE UMA EMPRESA DO RAMO FUMAGEIRO
}

\section{PREDICTIVE MAINTENANCE STRATEGY IN THE GRAPHICS DEPARTAMENT OF A TOBACCO COMPANY}

\author{
Guilherme Francez Toazza* E-mail: guilherme.toazza@souzacruz.com.br \\ Miguel Afonso Sellitto* E-mail: sellitto@unisinos.br \\ *Universidade do Vale do Rio dos Sinos (UNISINOS), São Leopoldo, RS
}

Resumo: O objetivo deste artigo foi descrever a implantação de uma estratégia de Manutenção Preditiva no departamento gráfico de uma empresa da indústria fumageira. O método de pesquisa foi a pesquisa-ação. Foram revisadas e usadas as técnicas de Analise de Vibração, Termografia, Ferrografia e Inspeção Sensitiva. Foi construída e aplicada uma estratégia de Manutenção Preditiva, integrada à estratégia de produção do departamento gráfico. Na pesquisa, foram levantados, no sistema de informação da empresa, o índice de quebra de equipamentos (downtime), custos com desperdícios de energéticos, custos de manutenção e disponibilidade de $\mathrm{H} / \mathrm{h}$ (homem/hora) e disponibilidade de equipamentos para a produção no período anterior a implantação de uma gestão de manutenção preditiva consistente e comparada com resultados após esta implantação. A partir dos resultados obtidos, ficou evidente que somente a manutenção preventiva, associada a um modelo parcial de manutenção preditiva, não foi suficiente para obter e manter a confiabilidade desejada em um departamento crítico para a empresa. A pesquisa concluiu que a implantação de uma estratégia de manutenção preditiva pode trazer resultados positivos em indicadores estratégicos que capturam objetivos compartilhados com outros setores da empresa, principalmente a produção.

Palavras-chave: Analise de Vibração. Termografia. Ferrografia. Inspeção Sensitiva. Manutenção Preditiva.

\begin{abstract}
The purpose of this article was to describe the implementation of a strategy for Predictive Maintenance in the graphics department of the company Souza Cruz SA after the techniques of Vibration Analysis, Thermography, Ferrography and Inspection Sensitive. The research method was the action-research. The proposed study is the application of a management Predictive Maintenance acting strategically within the context in which it is embedded. The article reviews the theoretical concepts and Predictive Maintenance techniques mentioned above. In the survey, were raised in the information system of the company, the rate of breakdown of equipment (downtime), waste energy costs, maintenance costs and availability of $\mathrm{H} / \mathrm{h}$ (man / hour) and availability of equipment for the production the period prior to the implementation of a predictive maintenance management and consistent compared with results after this deployment. From the results obtained, it was evident that only preventive maintenance, combined with a model predictive maintenance is not poor enough to maintain the desired reliability in a department of great importance to your company. So, it is possible to say that the implementation of a predictive maintenance strategy can make the Maintenance sector to work strategically with common goals to the company as a whole.
\end{abstract}

Keywords: Vibration Analysis. Thermography. Ferrography. Sensitive Inspection. Predictive Maintenance. 


\section{INTRODUÇÃO}

Atividades de manutenção têm assumido importante posição na estratégia de operações de empresas industriais (LIMA et al., 2010): seu gerenciamento estratégico pode contribuir para aumentar a eficiência geral da produção, ajudando a manter a disponibilidade de máquinas e reduzindo o custo por ociosidade (ABREU et al., 2013). Atividades de manutenção mal planejadas podem implicar quedas no volume de produção e atrasos nas entregas, eventualmente acarretando perda de vantagem competitiva na indústria (MENDES, 2011).

Atividades de manutenção podem ser classificadas segundo o tipo de intervenção: manutenção corretiva, que espera a falha para reparo ou substituição; manutenção preventiva, que se baseia em parâmetros de uso do equipamento, tais como tempo decorrido, quilometragem de veículos, ou número de ciclos de produção; manutenção preditiva que, por monitoramento das máquinas, torna possível a identificação do problema antes da ocorrência da falha; e manutenção proativa, que visa à identificação da causa-raiz dos problemas e não apenas dos sintomas que foram observados (BARKÓKEBAS JUNIOR et al., 2010).

Para este artigo interessa mais a manutenção preditiva. A manutenção preditiva inclui atividades que utilizem técnicas de monitoração de variáveis críticas em equipamentos. Tais variáveis devem informar sobre o estado do equipamento, seja sob a forma de inspeção sensitiva, seja sob a forma de instrumentação de campo (PERES e LIMA, 2008). Em manutenção preditiva, as informações adquiridas permitem identificar uma tendência no comportamento de um sistema produtivo ou de uma máquina isolada (RAMOS FILHO et al., 2010), permitindo prever o momento mais provável em que uma falha vai ocorrer e tomar uma decisão de intervenção antes da falha (SELLITTO et al., 2002). Uma estratégia de manutenção preditiva pode oferecer vantagens significativas em termos de qualidade do produto, segurança das operações, disponibilidade de sistemas e custos em operações industriais (BANA e COSTA et al., 2012). Durante a formulação da estratégia, decisões são necessárias, tais como a seleção e a priorização das técnicas que serão usadas para o diagnóstico precoce das falhas (CARNERO, 2005).

Geralmente, uma estratégia de manutenção preditiva exige um sistema informatizado de gestão e controle de atividades (PEREIRA et al., 2010). Uma 
estratégia de manutenção preditiva pode usar modelos otimizantes, balanceando intervalos entre inspeções e cálculo do momento ótimo para a troca de componentes em sistemas que se degradam ao longo do tempo (GRALL et al., 2002). A degradação pode ser devida a imperfeições na execução da manutenção, tal como ocorre em uma política de mínimos reparos (ZHOU et al., 2007) ou devida a imperfeições na formulação ou execução de políticas de manutenção preventiva (YOU et al., 2010). Em política de mínimos reparos, dada uma falha, a extensão e a duração do reparo são as menores possíveis, de modo que o sistema volte a operar em curto espaço de tempo. Neste caso, modelos otimizantes podem considerar também a extensão do reparo como variável de decisão (ZHOU et al., 2007).

Em particular, interessa estudar a prática da manutenção preditiva em uma empresa do setor fumageiro, a Souza Cruz S/A. Líder do mercado nacional de cigarros e integrante do grupo British American Tobacco (BAT), a Souza Cruz S/A opera em toda a cadeia produtiva, incluindo a produção de sementes, o processamento de fumo, a fabricação e distribuição direta de cigarros em mais de 240 mil pontos de venda. A empresa entende que o cenário de negócios exige o cumprimento de prazos e metas cada vez mais desafiadores, fazendo com que as atividades mais críticas que possam impactar no processo e nos resultados sejam exploradas e otimizadas.

O objetivo deste trabalho é descrever a implantação e a execução de uma estratégia de Manutenção baseada em procedimentos preditivos no departamento gráfico de uma empresa do ramo fumageiro. O objeto de estudo foi o Departamento Gráfico (DG) da empresa. O método de pesquisa foi a pesquisa-ação. As principais técnicas de pesquisa foram: análise de documentos; consultas ao sistema de informação da manutenção; entrevistas com gestores da manutenção; observação participante; e intervenção. Os objetivos específicos foram: (i) descrever a situação inicial em que se encontravam as práticas até então adotadas de manutenção preditiva no DG; (ii) descrever os procedimentos de implantação e organização da manutenção preditiva no DG, referentes às técnicas de inspeção sensitiva, analise de vibração, termografia e ferrografia; e (iii) apresentar e discutir os principais resultados numéricos da implantação. 
O restante do artigo está organizado em: revisão bibliográfica; descrição da pesquisa; discussão dos resultados; e considerações finais. Parte da pesquisa foi financiada pelo CNPq.

\section{MANUTENÇÃO PREDITIVA}

Intervenções planejadas de manutenção têm se mostrado uma estratégia eficaz, mesmo que, ao longo do tempo, sua prática reduza a vida média de uso de componentes e subsistemas. Como estes são trocados em intervalos fixos, definidos por algum tipo de critério, existe uma possibilidade importante de troca precoce, enquanto o item ainda é útil, o que acarreta atividades desnecessárias e introduz riscos na operação, pois não se sabe se o novo componente será mais robusto do que o componente que sai (SWANSON, 2003). Por outro lado, aumentar o intervalo fixo de troca também aumenta a probabilidade de falha inesperada e pode requerer atividades extraordinárias, não-programadas de reparo, o que pode ser tão danoso quanto a primeira situação (CHINESE e GHIRARDO, 2010).

Um avanço importante em relação às trocas em intervalos fixos pode ser a adoção de técnicas de medição direta baseadas em sensoriamento, capazes de informar acerca da situação de uso do item, antes da decisão de troca (HASHEMIAN e BEAN, 2011). Com tal procedimento, reduções significativas de custo podem ser obtidas, que geralmente compensam o investimento necessário em equipamentos, sistemas de medição e gerenciamento de resultados da medição. Havendo disponibilidade de equipamentos e sistemas de gerenciamento, é possível adotar uma estratégia de manutenção preditiva, cujo principal papel é estimar, por modelos baseados em medições, o tempo mais provável até a próxima falha e assim planejar intervenções de correção compatíveis com estes tempos (AHMAD et al., 2011).

Em suma, estratégias baseadas em técnicas de manutenção preventiva consistiam originalmente na reposição ou reparo programado, ou seja, préagendado de componentes e subsistemas (SELLITTO, 2005). Tais técnicas evoluíram para o atual formato de atividades conhecido como manutenção preditiva, na qual perdas dos equipamentos são monitoradas antes que se tornem quebras, podendo assim ser evitadas por intervenções planejadas (SOLA et al., 2006). A manutenção preditiva pode ser utilizadas em conjunto com iniciativas vinculadas à 
Manufatura Enxuta (DURAN e BATOCCHIO, 2003) ou como apoio à Manutenção Centrada em Confiabilidade (FERREIRA et al., 2010). A confiabilidade de um equipamento é a probabilidade de que este desempenhe a função requerida, por um intervalo de tempo estabelecido, sob condições definidas de uso (SELLITTO et al., 2002).

A manutenção preditiva baseia-se na leitura de variáveis críticas cujos limites são previamente definidos. Quando as leituras ou a projeção por modelos se aproximam destes limites, uma intervenção de manutenção deve ser planejada, de modo a controlar a falha iminente (GARG e DESHMUKH, 2006). Para tanto, sistemas instrumentados de aquisição e monitoramento de dados em tempo real podem ser necessários (BEVILACQUA e BRAGLIA, 2006). A detecção antecipada de uma anomalia permite um diagnóstico precoce da falha e possibilita a execução de um plano de ações corretivas, no momento e na intensidade adequados ao tipo de falha. Do mesmo modo, a contínua monitoração de certas variáveis permite que se trace e acompanhe um perfil ao longo do tempo da condição de uso ou de deterioração do equipamento (GARCIA et al., 2006).

Algumas das técnicas mais usadas em estratégias de manutenção preditiva são a inspeção sensitiva, a análise de vibração, a termografia e a ferrografia.

A inspeção sensitiva utiliza os sentidos humanos para detecção e diagnósticos de potenciais falhas. Em alguns casos, utilizam-se ferramentas como auxílio durante a inspeção, tais como amplificadores e aparelhos de ultrassom. É possível identificar concentração de gases pelo olfato (SCHIRMER et al., 2007), temperatura e vibração por apalpação (OTANI e MACHADO, 2008) e quebras por inspeção visual e fotográfica (MIRSHAWKA, 1991).

A análise de vibração pode detectar os níveis de vibração, ruído, desbalanceamento e temperatura em equipamentos, sem seu desligamento. A principal variável observada é a frequência de ressonância, medida em Hertz $(\mathrm{Hz})$. Cada componente produz uma vibração com frequência específica que, em condições normais de funcionamento, alcança uma amplitude máxima determinada (YA'CUBSOHN, 1983). Caso este padrão se modifique, é possível que haja algum problema em andamento (NEPOMUCENO, 1985). A análise das vibrações permite detectar falhas potenciais e revelar suas origens, com antecedência suficiente para 
definir o momento mais oportuno para intervenções corretivas (TACQUES et al., 2009). Esta prática é conhecida como manutenção sob condição.

A termografia usa instrumentos sensíveis à radiação infravermelha para, sem contato físico, visualizar o perfil térmico e medir as variações do calor emitido pelas diferentes regiões da superfície de um corpo (FLÓRIO FILHO et al., 2006). Com isto, é possível avaliar o estado de um equipamento sem seu desligamento (SANTOS e PEREIRA, 2010), pois anomalias observadas nas condições térmicas são sinais que antecipam possíveis problemas em equipamentos (TSANG, 1995). A termografia infravermelha (TIV) se baseia no mapeamento térmico de um componente para a localização de suas regiões danificadas ou defeituosas ou ainda submetidas a esforços ou atritos excessivos (TARPANI et al., 2009). Em equipamentos elétricos, falhas em redes de transmissão, distribuição, subestações e centros de carga podem ser prevenidas com o auxílio da termografia (MIRSHAWKA, 1991).

A ferrografia é uma técnica de avaliação das condições de desgaste de componentes por meio da quantificação e observação das partículas em suspensão no lubrificante (NOGUEIRA e REIS, 2010). A ferrografia determina a severidade, modos e tipos de desgaste em máquinas por meio da identificação da morfologia, acabamento superficial, coloração, natureza e tamanho das partículas encontradas em amostras de óleos ou graxas lubrificantes de qualquer viscosidade, consistência e opacidade (KARDEC et al., 2002). Um dos benefícios que a ferrografia oferece é a determinação com maior acuracidade do momento correto para a troca do lubrificante: troca antecipada é desperdício; troca retardada coloca em risco outros componentes (VIEIRA et al., 2007). A ferrografia quantitativa quantifica partículas grandes e pequenas, permitindo controlar a contaminação do meio oleoso. Já a ferrografia analítica permite classificar as partículas originadas do desgaste (NOGUEIRA e REIS, 2010). Segundo Souza (2000), é possível analisar o aspecto e o tamanho das partículas presentes no óleo, permitindo identificar com precisão o nível de desgaste. A ferrografia também é capaz de identificar metais não-ferrosos, outros contaminantes sólidos inorgânicos, tais como areia, fibras ou sais e produtos originados da degradação do próprio lubrificante, tais como borras, gel, lacas ou vernizes (OTAMI e MACHADO, 2008). 


\section{A PESQUISA}

A pergunta de pesquisa foi: como implantar uma estratégia de manutenção preditiva em uma planta fabril e que resultados podem ser esperados desta implantação? O método de pesquisa foi a pesquisa-ação. A pesquisa-ação visa obter melhorias em processos ou produtos aproveitando conhecimentos existentes e ao mesmo tempo produzir novos conhecimentos sobre o objeto de pesquisa (FRENCH, 2009). Em pesquisa-ação, o conhecimento é produzido a partir da solução de um problema prático em uma atividade de campo, com objetivos e problemas reais (ALTRICHTER et al., 2002). O objeto de estudo foi a Empresa de Tabacos Souza Cruz. Em particular, foi estudada a instalação de Cachoeirinha, RS, e dentro desta, o Departamento Gráfico (DG). A principal contribuição científica da pesquisa foi o método usado para diagnosticar a necessidade, implantar a estratégia de manutenção preditiva, os resultados específicos da ação e o tipo de resultados que podem ser esperados de tal implantação.

A Fábrica de Cachoeirinha é a fábrica mais moderna da empresa e uma das mais modernas e competitivas no mundo, empregando tecnologias e processos produtivos atualizados. Produz em média 30 bilhões de cigarros por ano (em torno de $40 \%$ do volume fabricado pela empresa). As principais áreas da fábrica são o Processo Primário (PMD), responsável pelo processamento de fumos e o Processo Secundário (SMD), responsável pela fabricação de cigarros. Estas áreas são suportadas por estrutura administrativa composta por: área de engenharia, setor de qualidade, área de treinamento e equipe de EH\&S (segurança e meio ambiente). A instalação também sedia o Centro de Pesquisas, responsável pela coordenação regional de desenvolvimento de produtos para toda região das Américas e Canada.

O DG, assim como a empresa, é certificado desde 2005 por um sistema integrado denominado QuEnSH (Quality, Environment, Safety, Health - Qualidade, Ambiente, Segurança, Saúde Ocupacional), desde então renovado anualmente. Em 2005, a Souza Cruz concluiu o processo de certificação integrada, tornando-se pioneira no grupo British American Tobacco a obter para sua cadeia produtiva certificação simultânea das normas internacionais de Qualidade (ISO 9001), Meio Ambiente (ISO 14001) e Segurança e Saúde Ocupacional (OHSAS 18001). As atividades certificadas se estendem desde a assistência técnica dada ao produtor de 
fumo até a entrega nos pontos de venda. A empresa se estrutura em: três centros de processamento de fumo (Santa Cruz do Sul, Blumenau e Rio Negro), unidades industriais em Cachoeirinha e Uberlândia (fábricas, Departamento Gráfico e Centro de Pesquisas e Desenvolvimento) e Distribuição, englobando seis Centrais Integradas de Distribuição, em Belo Horizonte, Curitiba, Porto Alegre, Recife, Rio de Janeiro e São Paulo.

O DG estudado, de Cachoeirinha, conta com equipamentos de ponta que possibilitam a impressão de embalagens com maior número de cores. Seus equipamentos são críticos e podem ser considerados gargalos: a parada dos equipamentos do DG representa a parada de linhas de produção, tal como usualmente ocorre em instalações fabris pertencentes a indústrias de processamento contínuo (SELLITTO, 2002). O DG é responsável por cerca de 95\% da produção de rótulos, estojos, envoltórios, reforços, forros impressos e ponteiras (com perfuração a laser), imprimindo cerca de 200 milhões de $\mathrm{m}^{2}$ por ano.

O método de trabalho foi:

- revisão bibliográfica dos conceitos de manutenção preditiva, analise de vibração, termografia, ferrografia e inspeção sensitiva;

- por observação participante e intervenção, foi feito um levantamento prévio da situação estratégica da manutenção preditiva no DG;

- por entrevistas com gestores, observação participante e intervenção, foi feita a implantação da gestão de manutenção preditiva no departamento gráfico; e

- por análise de documentos e consultas ao sistema de informação da manutenção, foi feito levantamento histórico de paradas de máquinas por manutenção corretiva (downtime), disponibilidade de máquina, disponibilidade de $H / h$, atrasos na entrega de relatórios de manutenção preditiva, custo de manutenção e custo com energéticos do objeto de estudo.

\subsection{Situação Anterior: Práticas de Manutenção Preditiva}

A descrição que segue é baseada em observação participante e nãoparticipante e entrevistas não-estruturadas com gestores das atividades de manutenção do DG. Nesta sessão alcança-se o primeiro objetivo específico. 
A estratégia de Manutenção Preditiva originalmente existente incluía análise de vibração, ferrografia e termografia. Estudos foram feitos a partir do acompanhamento e observação da rotina do setor de Planejamento e Controle de Manutenção (PCM) do departamento gráfico.

A técnica de Análise de Vibração foi a primeira a ser observada. Relatórios referentes às analises eram entregues um dia antes da realização da manutenção preventiva, dificultando o planejamento da correção dos pontos críticos detectados, que até então eram tratados somente durante o período de manutenção preventiva. Além do atraso na entrega dos relatórios, tal técnica dificilmente cobria $100 \%$ dos componentes que deveriam ser monitorados, deixando pontos importantes do parque gráfico a descoberto. Foi possível observar que indicadores não eram atualizados periodicamente, nem havia planos de ação para tais indicadores.

Inspeções termográficas eram programadas por outro departamento, o setor secundário (SMD). Este planejamento apresentava constantes falhas, pois a divisão física e descentralização da gestão técnica geravam conflitos com outras atividades de rotina. Desde 2009 (inauguração do DG no RS) até metade de 2012 foi emitido apenas um relatório de termografia. Diversos desarmes de máquinas ocorriam durante a produção, devido a pontos de aquecimento que poderiam ter sido identificados e evitados a partir de atividades preventivas oriundas de recomendações de relatórios de inspeção termográfica.

Apesar de existirem análises ferrográficas, ocorriam substituições aleatórias de óleos lubrificante. Observações foram feitas, nas quais foi possível perceber desperdícios de enérgicos como vapor e ar comprimido. Após acompanhamento, dados e resultados das observações foram divulgados aos principais gestores do departamento gráfico por exposição visual embasada em dados históricos.

A necessidade de gestão e reformulação da estratégia existente de Manutenção Preditiva ficou explícita neste ponto.

\subsection{Implantação: Gestão das Práticas de Manutenção Preditiva}

A descrição que segue é baseada em observação participante e intervenção e também em consultas ao software de gestão de manutenção da empresa (SAP). Nesta sessão alcança-se o segundo objetivo específico. 
O atraso na entrega de relatórios, coleta de dados e diagnósticos foi o ponto determinante para motivar a reestruturação da prática preditiva de manutenção. A confiabilidade da manutenção diminuía ao ponto que a equipe não atendia ao mínimo desejado devido à sobrecarga de atividades. No período de 2009 até parte do segundo semestre de 2012, a manutenção preditiva era composta por um analista, responsável pela análise de vibração e ferrografia, e um técnico de manutenção eletrônica, responsável pela termografia. Os colaboradores eram responsáveis por: coletar e analisar os dados; emitir relatórios; sugerir melhorias; gerar histórico manual de falha e correções; criar e atualizar indicadores; criar planos de ação; acompanhar manutenções em pontos levantados após inspeções de preditiva; garantir sobressalentes que atendam de forma coerente o histórico de falha. Ou seja, o foco não estava na análise e sim na tarefa.

A partir do segundo semestre de 2012, foi criada a função inspetor de rota, com a admissão de dois colaboradores, e incorporadas as seguintes atribuições à equipe: criação de notas Z2; criação de rotas de coleta de dados e histórico no sistema de informação de manutenção; gerar requisitos de adequação de segurança; e integrar os resultados com o sistema de RCM (Reliability Centered Maintenance - Manutenção Centrada em Confiabilidade) existente na empresa. Nas notas Z2, as falhas iminentes levantadas durante as análises são registradas no software de gestão de manutenção da empresa. Foi criada a função Inspetor de Rota, o que propiciou, além da aplicação das técnicas de análise de vibração, ferrografia e termografia, a inclusão da técnica da inspeção sensitiva na linha do sistema de ar comprimido e linha de vapor. $\mathrm{O}$ analista passou a dedicar-se mais à análise de dados, que passaram a ser coletados por dois inspetores de rota com formação técnica em mecânica.

A Figura 1 apresenta o novo de fluxo de informações e gestão dos dados coletados durante as inspeções preditivas. 
Figura 1- Fluxo de informação da Manutenção Preditiva

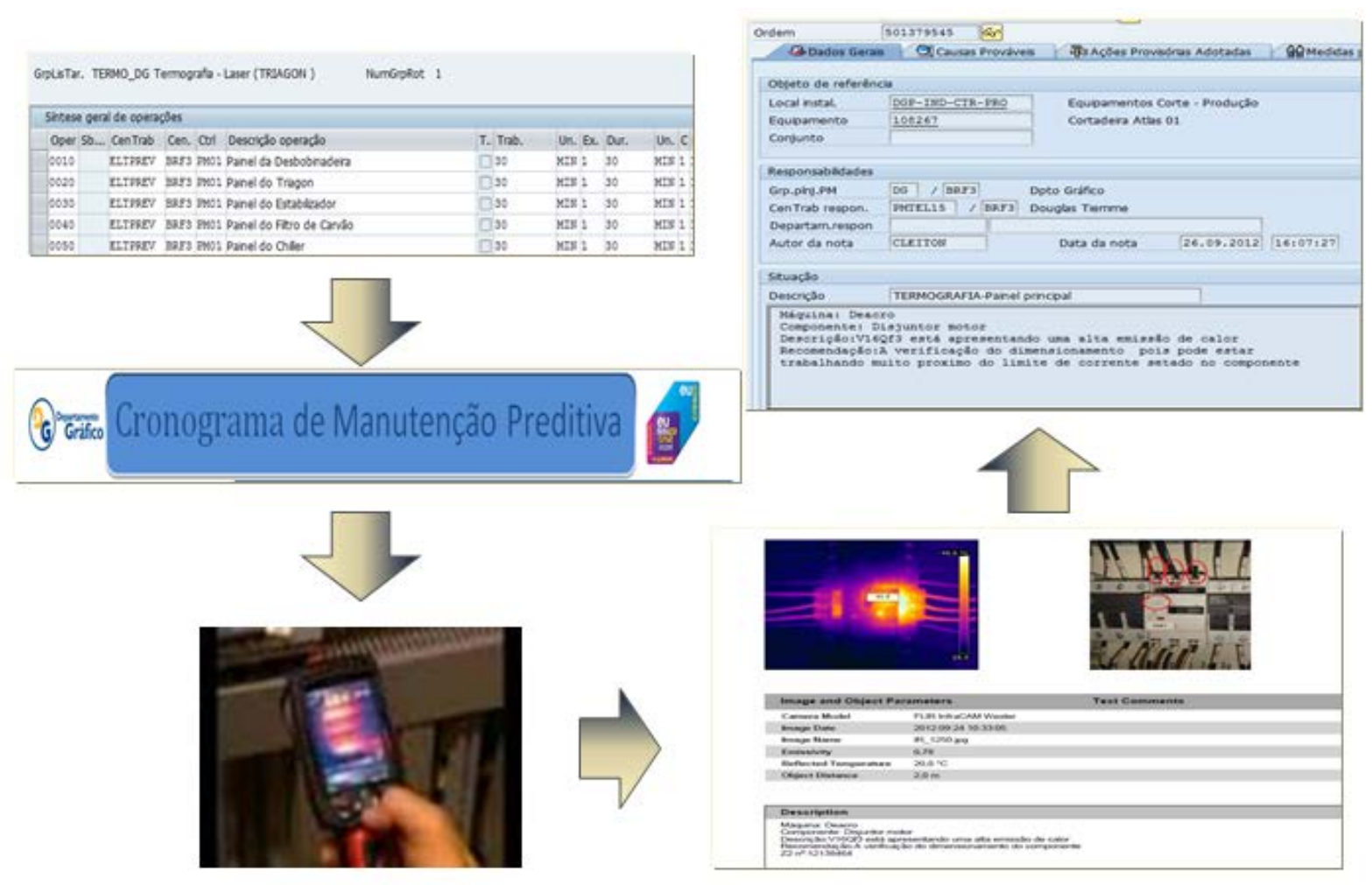

Fonte: sistema de informação da empresa

O sistema de informação gera a rota de coleta e o cronograma de inspeções, revisados de acordo com o histórico dos dados coletados. Os dados coletados são armazenados e analisados e um programa de intervenções em equipamentos e de segurança é gerado, completando o ciclo da figura. A principal contribuição desta etapa foi a criação de rota e cronograma de inspeções. A solicitação de inspeção contém o tempo e os procedimentos necessários, o que permite planejar a capacidade dos inspetores de rota.

O encerramento das ordens é de responsabilidade do próprio inspetor, que deve descrever os valores encontrados na determinada inspeção.

A Figura 2 mostra um exemplo de cronograma de Manutenção Preditiva. 
Figura 2 - Cronograma de Manutenção Preditiva

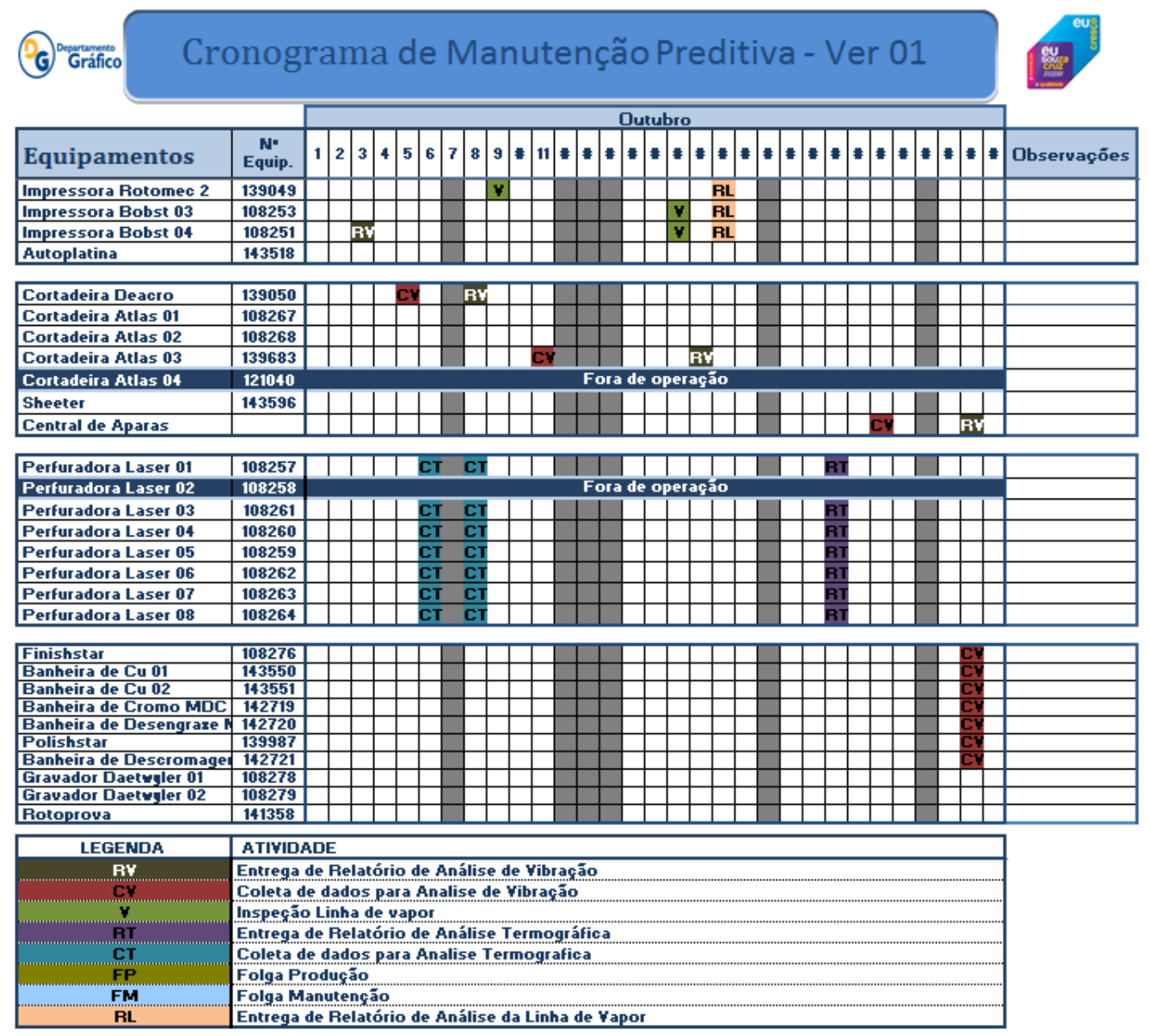

Fonte: sistema de informação da empresa

A Figura 3 mostra um exemplo de Plano de Coleta de Dados para um determinado equipamento com periodicidade de 544h (um mês): mensalmente, uma ordem de inspeção preditiva é gerada. 
Figura 3 - Plano de coleta de dados para Análise de Vibração

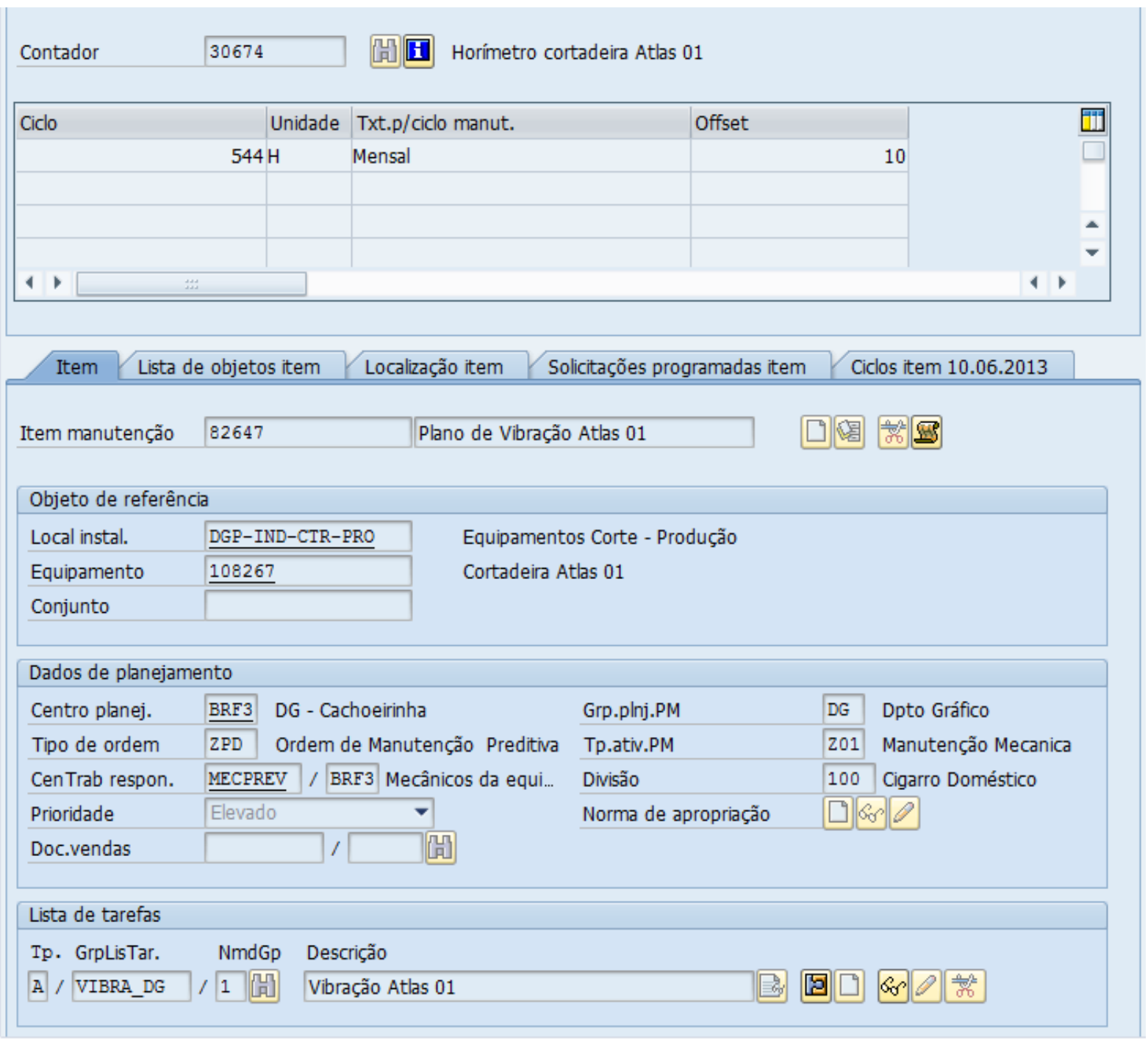

Fonte: Software de gestão da empresa

Feita a análise de vibração, pode-se emitir o diagnóstico dos pontos analisados e as recomendações de manutenção. A Figura 4 traz um exemplo de relatório emitido após a análise de vibração, contendo o diagnostico da medição, histórico de medições anteriores e recomendações. 
Figura 4 - Relatório após análise de vibração

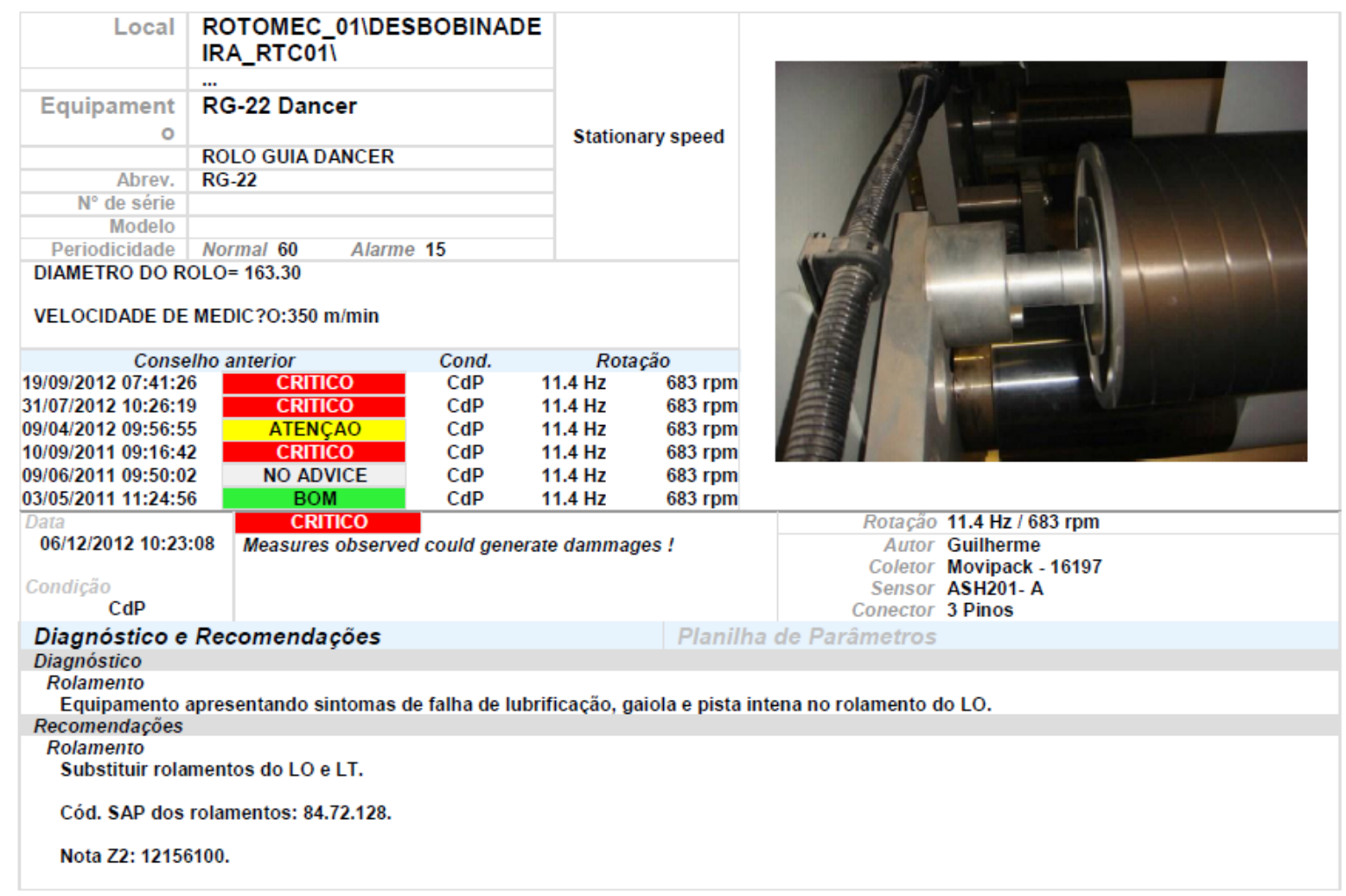

Fonte: sistema de informação da empresa)

Além da criação e emissão de relatórios contendo os pontos medidos e analisados, adotaram-se as seguintes medidas: emissão e divulgação de relatórios gerenciais mostrando por equipamento o percentual de atendimento ao plano de analise; percentual de atendimento às notas Z2; efetividade das recomendações descritas nas notas Z2; índice de quebras por equipamento; status mensais da atividade; e acompanhamento das falhas corrigidas.

O percentual de atendimento ao plano de manutenção preditiva refere-se ao que de fato foi executado, conforme solicitação do cronograma de manutenção preditiva que foi feito a partir das ordens geradas automaticamente. O indicador de atendimento à nota Z2 tem como objetivo medir a eficiência na tratativa das notificações (notas Z2) abertas a partir de detecções de falha iminente. A efetividade das recomendações é medida após o trabalho de correção do componente com falha iminente. Recomendação efetiva deve condizer com a informação descrita no relatório de recomendações. Cada componente pertencente ao plano de manutenção preditiva que quebra sem detecção antecipada é considerado um item 
que quebrou por falha da Manutenção Preditiva. O indicador que mede estas falhas é o Índice de Quebras.

Para resumir a leitura, usa-se o indicador de Status Mensais. Este indicador mostra o número de componentes medidos separados por seus diagnósticos: bom (verde); atenção (amarela); critico (vermelha). Componentes diagnosticados como bons não apresentam nenhum tipo de iminência de falha e trabalham próximo da capacidade nominal. Nesse caso, não é necessário gerar recomendação. O diagnóstico de atenção indica que o componente apresenta principio de falha. $A$ recomendação, geralmente, é acompanhar o equipamento onde está o componente até a próxima medição. Componente crítico representa iminência de falha. Recomenda-se, então, a programação na próxima oportunidade factível, para a manutenção sob condição. Caso não haja oportunidade no horizonte de tempo exigido, a recomendação é de parada no equipamento para reparo, antes que se configure uma falha de emergência.

O tipo de falha percebido nas análises é mostrado pelo indicador de Acompanhamento das Falhas. Além do relatório técnico contendo as informações a partir das medições em campo, é gerado um relatório para cada inspeção. É o que mostra a Figura 5. 
Figura 5 -Relatório Gerencial

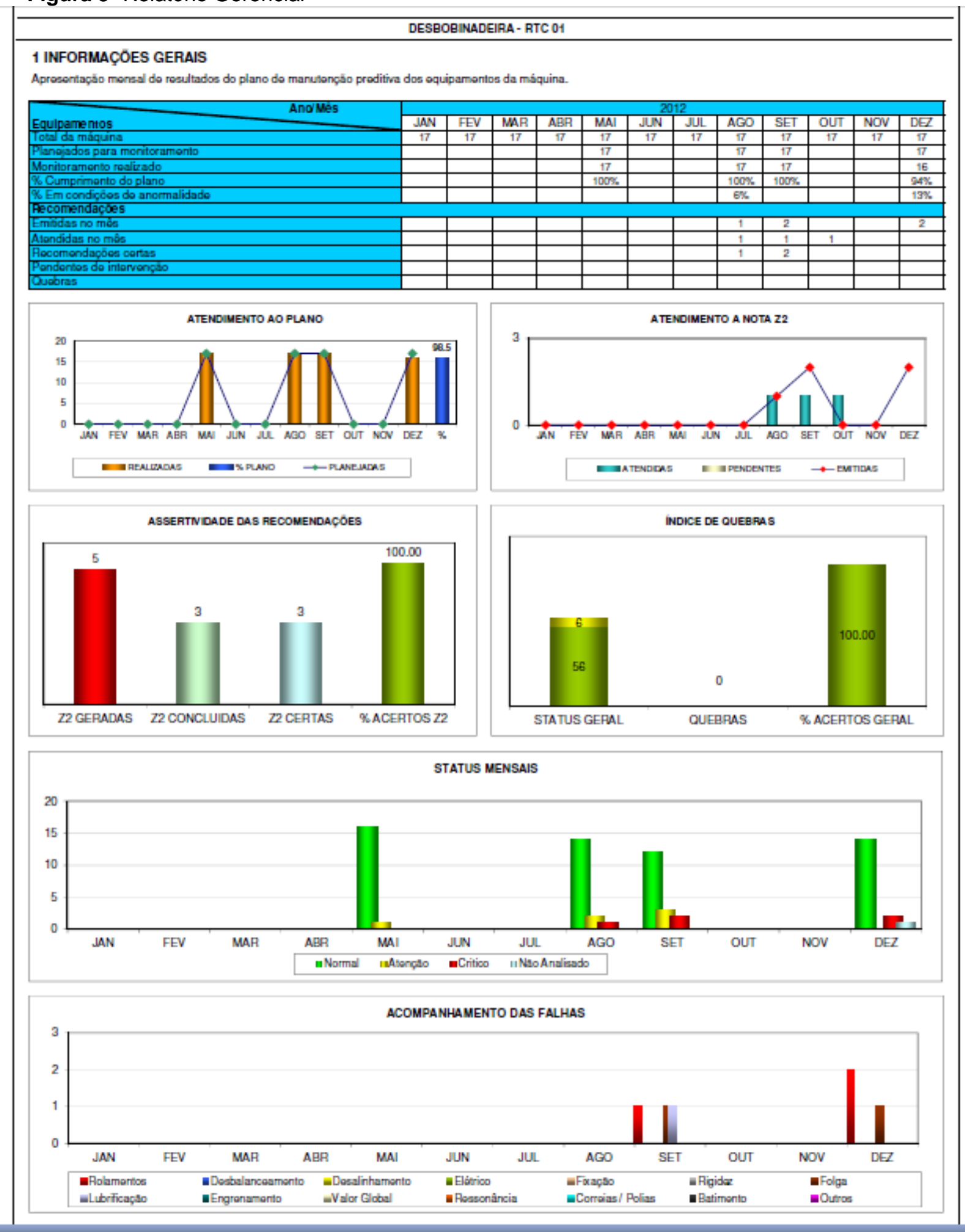

Fonte: Sistema de informação da empresa

Além das inspeções e diagnósticos, foi possível implantar a inspeção sensitiva. Nesse caso, a inspeção sensitiva vale-se de aparelho ultrassom como auxílio para detectar falhas e desperdícios de ar comprimido e vapor. Esta prática é

Revista Produção Online, Florianópolis, SC, v.15, n. 3, p.783-806, jul./set. 2015. 
utilizada na unidade da Souza Cruz em Uberlândia, MG. Por meio de atividades de benchmarking, foi possível perceber, que apesar da simplicidade da prática, é possível alcançar resultados satisfatórios no âmbito financeiro e operacional.

O relatório mostrado na Figura 6 compila as informações obtidas após a inspeção nos purgadores da linha de vapor.

Figura 6 - Relatório dos purgadores
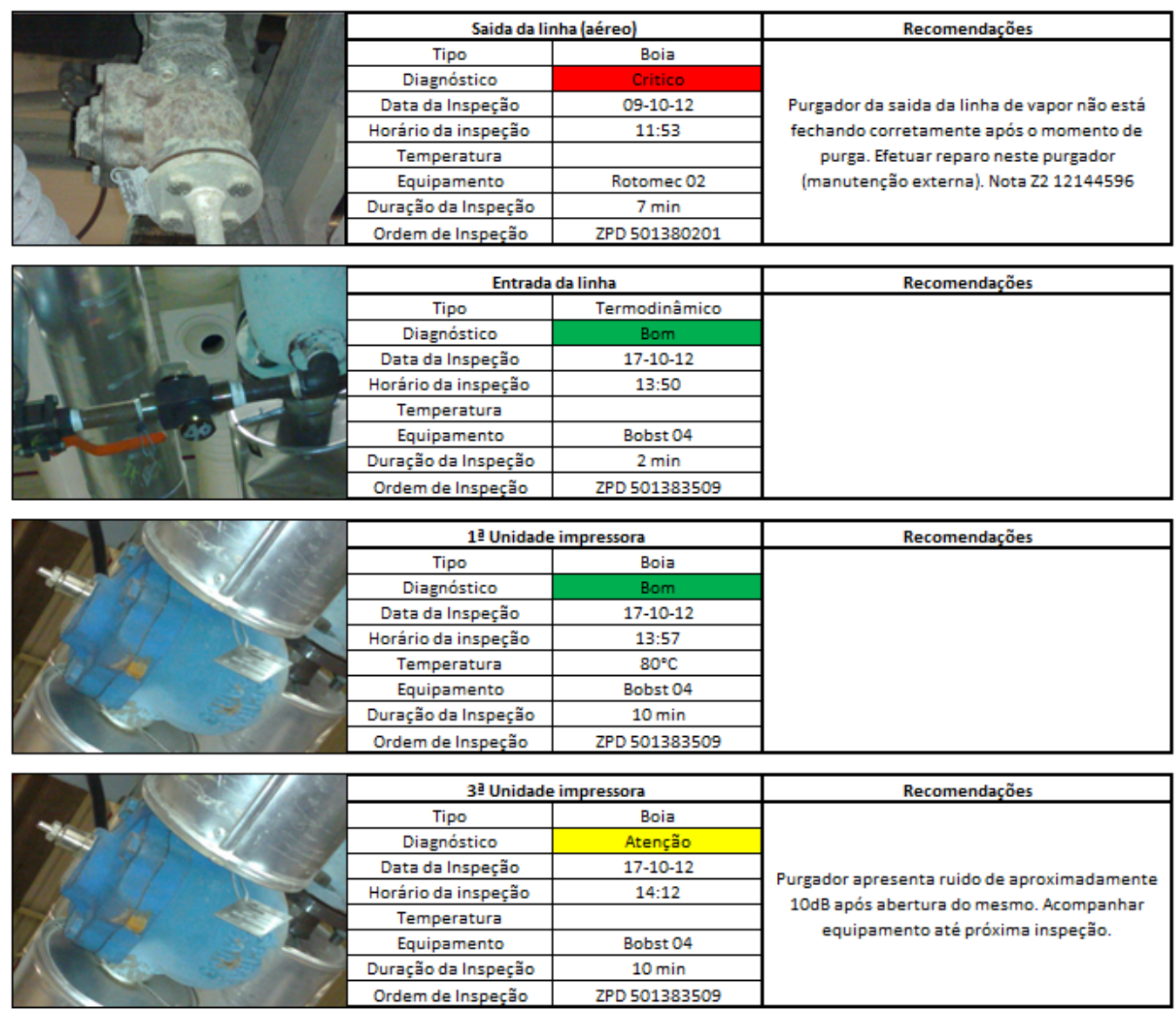

Fonte: Sistema de informação da empresa

Neste relatório, deve constar o tipo de purgador (boia ou termodinâmico); o diagnóstico informado pelo analista; data da inspeção; temperatura que a estufa de aquecimento da secagem da tinta próxima ao purgador inspecionado se encontra no momento da medição; a denominação do equipamento; duração da inspeção, que se trata do tempo que o inspetor teve que esperar até que o purgador atuasse; ordem que solicitou a inspeção; recomendação, contendo o número da notificação (nota Z2) solicitando a intervenção, se necessário. 


\section{DISCUSSÃO DOS RESULTADOS}

Nesta sessão alcança-se o terceiro objetivo específico.

Apesar de se tratar de um período de apenas doze meses, foi observada evolução positiva em vários aspectos da estratégia de manutenção. Essa observação ocorreu a partir de melhorias na operação, cujos resultados foram mensurados e processados pelo sistema de informações da empresa.

Um resultado evidenciado foi a redução de custos com o consumo de ar comprimido devido à introdução da inspeção sensitiva. A última medição antes da introdução da inspeção sensitiva (7/12) apontou um consumo médio diário por vazamentos de $530 \mathrm{Nm} 3 / \mathrm{h}$, gerando um custo médio diário de $\mathrm{R} \$ 407,00$. A medição é feita ligando-se os compressores por dez minutos antes do início da produção. Todo o consumo registrado nesta situação é devido a vazamentos. Após seis meses (12/12), 84\% dos vazamentos mapeados na inspeção haviam sido removidos e os consumos e custos médios diários por vazamentos foram respectivamente de 430 $\mathrm{Nm}^{3} / \mathrm{h}$ e $\mathrm{R} \$ 338,00$. A redução não foi proporcional à fração de vazamentos eliminadas porque novos vazamentos surgiram em locais já inspecionados. Observa-se então que a frequência de inspeção deve ser maior do que a atual, o que, por ora, ainda não é possível, dada a quantidade de inspetores disponíveis. Por ora, a periodicidade é trimestral.

Outro resultado observado foi a redução de cerca de $30 \%$ no consumo unitário de vapor, em doze meses (3/12 a 3/13). O consumo médio diário passou de 29,64 para 23,37 toneladas de vapor. Vazamentos nos flanges e conexões foram detectados pela inspeção sensitiva, apoiada por ultrassom, e corrigidos a partir de ordens de serviço emitidas pelo sistema de informação. O uso de ultrassom permite a amplificação do ruído de purgadores em atuação: ruído em excesso indica que o purgador permanece parcialmente aberto quando deveria estar fechado. O incorreto funcionamento de purgadores acarreta acréscimo de consumo de vapor.

Outro resultado observado foi a redução de cerca de $35 \%$ no tempo de máquina desligada (downtime) da impressora Rotomec 01, a principal impressora do DG, por paradas mecânicas. O percentual de tempo desligado passou de 3,8\% (12/11) para 2,5\% (12/12), o que representou em média cerca de 50 toneladas de produto acabado a mais no ano. Quanto a paradas eletrônicas, o percentual de 
tempo desligado passou de 4,4\% (12/11) para 3,5\% (12/12), o que representou em média cerca de 40 toneladas de produto acabado. A principal causa da melhoria foi a adoção da prática de manutenção baseada em condição, ou seja, inspeções preditivas e intervenções de correção. A impressora Rotomec 01 é composta por 192 rolos, 26 motores, 8 mancais e 20 exaustores que foram incluídos no plano de manutenção preditiva. Com a detecção antecipada de falhas nestes componentes, foi possível o planejamento para execução da manutenção sob condição, antes das quebras.

Outro resultado observado foi a diminuição do número global de atividades e por consequência do custo de manutenção corretiva e preventiva. A adoção da análise de vibração permitiu que o plano de manutenção de preventiva fosse reduzido, ou seja, itens que eram substituídos periodicamente deixaram de sê-lo, passando a ser objeto de manutenção sob condição. Por exemplo, na Rotomec 1, as substituições periódicas envolviam 24 subconjuntos, com período médio de troca de 2.500 horas. Após um ano (de janeiro a dezembro de 2013), o período médio de troca baseada em manutenção preditiva passou para 4.750 horas, ou seja, o intervalo entre as trocas em média agora é quase o dobro do anterior. Do mesmo modo, após a implantação da inspeção termográfica, houve diminuição das atividades descritas no plano de manutenção preventiva eletrônica. Atividades, tais como reaperto de conexões do painel elétrico, foram substituídas por manutenção sob condição a partir da inspeção termográfica. Tal mudança significa que só os pontos que apresentarem aquecimento detectados pela termografia serão reapertados ou redimensionados.

Por fim, foi observada redução no consumo de óleo lubrificante, devido à adoção da análise por ferrografia. Foi possível observar que a substituição periódica de lubrificantes era exagerada em dois equipamentos centrais: as impressoras Bobst 03 e 04. Juntas, estas duas impressoras produzem 50\% do volume mensal do DG. Para os demais equipamentos, observou-se que a prática de análise de óleo não era viável devido à baixa quantidade de lubrificante que estes demais equipamentos requerem. A estratégia de manutenção preventiva existente até a metade de 2012 contemplava a substituição de forma semestral (orientação dada por fabricante do equipamento) dos lubrificantes. A partir de junho de 2012, a técnica de ferrografia aboliu a troca periódica destes lubrificantes. 
Durante o período de um ano (1/13 a 12/13), caso ocorressem as trocas planejadas conforme a estratégia anterior, haveria um custo esperado de $\mathrm{R} \$$ 34.991,20. No período, foram canceladas várias trocas que anteriormente teriam sido feitas, pois análises indicaram que as condições do óleo de ambas as máquinas se mantiveram dentro dos padrões estabelecidos pelo fabricante e, portanto aceitáveis pela manutenção. Com isso, foi possível evitar um total de 96 horas na Bobst 03 e 144 horas na Bobst 04 de indisponibilidade para a produção, o que representou cerca de 120 toneladas de produto a mais que foi entregue no período.

\section{CONSIDERAÇÕES FINAIS}

O objetivo deste artigo foi descrever a implantação de procedimentos de manutenção preditiva dentro do departamento gráfico de uma empresa do ramo fumageiro. O método de pesquisa foi a pesquisa-ação em um departamento de uma empresa da indústria fumageira. A principal contribuição científica da pesquisa foi o método usado para diagnosticar a necessidade, implantar a estratégia de manutenção preditiva e o tipo de resultados que podem ser esperados de tal implantação. Tais contribuições foram apresentadas nos capítulos 3 e 4 deste artigo. Os dados empregados no estudo se originaram do histórico de manutenção gerenciado pelo setor de Planejamento e Controle de Manutenção (PCM). A acurácia das informações contidas no banco contribuiu para o estudo.

O departamento estudado contém equipamentos críticos e com tecnologia complexa, podendo ser considerado um gargalo da empresa, pois sua interrupção causa interrupção nas linhas de produção. Sendo um processo contínuo, falhas e paradas não programadas de qualquer equipamento do departamento pode impactar negativamente em toda a companhia, como descrito em Sellitto (2002).

As práticas de Manutenção Preditiva evidenciadas no estudo têm possibilitado prever com suficiente acurácia a ocorrência de falhas em componentes ou subsistemas. Desta forma, é possível planejar paradas de máquina em momentos oportunos para a correção ou prevenção da falha, segundo uma estratégia de manutenção sob condição. As técnicas de manutenção preditiva que foram implantadas têm contribuído para evitar 0 desperdício com atividades desnecessárias de manutenção, relativas a componentes que não necessitariam de 
troca ou reparo, mas na estratégia anterior eram trocados incondicionalmente. Tal prática resulta em maior disponibilidade do equipamento para produção e em diminuição dos custos de produção.

Como continuidade de pesquisa, sugerem-se mais estudos de caso referente a implantações de estratégias de manutenção preditiva, de modo a caminhar em direção a uma teoria que possa ajudar a explicar as características, os benefícios e as eventuais desvantagens de estratégias de manutenção de equipamentos industriais baseadas em técnicas preditivas.

\section{REFERÊNCIAS}

ABREU, J.; MARTINS, P.; FERNANDES, S.; ZACARIAS, M. Business Processes Improvement on Maintenance Management: A Case Study. Procedia Technology, v.9, n.2, 320-330, 2013. http://dx.doi.org/10.1016/j.protcy.2013.12.036

AHMAD, R.; KAMARUDDIN, S.; AZID, I.; ALMANAR, I. Maintenance management decision model for preventive maintenance strategy on production equipment. Journal of Industrial Engineering International, v.7, n.13, p.22-34, 2011.

ALTRICHTER, H.; KEMMIS, S.; MCTAGGART, R.; ZUBER-SKRRIT, O. The Concept of action research. The Learning Organization, v.9, n.3, p.125-131, 2002.

http://dx.doi.org/10.1108/09696470210428840

BANA E COSTA, C.; CARNERO, M.; OLIVEIRA, M. A multi-criteria model for auditing a Predictive Maintenance Programme. European Journal of Operational Research, v.217, n.2, p.381-393, 2012. http://dx.doi.org/10.1016/j.ejor.2011.09.019

BARKÓKEBAS JUNIOR, B.; VASCONCELOS, B.; MONTEIRO, M.; MACEDO, D.; RAMOS, A. Ensaios Não Destrutivos por Liquido Penetrante como Ferramenta de Auxilio à Manutenção Preditiva de Equipamentos de Elevação da Construção Civil, Anais do XXX ENEGEP - Encontro Nacional de Engenharia de Produção. São Carlos: ABEPRO, 2010.

BEVILACQUA, M.; BRAGLIA, M. The analytic hierarchy process applied to maintenance strategy selection. Reliability Engineering and System Safety, v.70, n.1, p.71-83, 2000. http://dx.doi.org/10.1016/S0951-8320(00)00047-8

CARNERO, M. Selection of diagnostic techniques and instrumentation in a predictive maintenance program. A case study. Decision Support Systems, v.38, n.4, p.539-555, 2005.http://dx.doi.org/10.1016/j.dss.2003.09.003

CHINESE, D.; GHIRARDO, G. Maintenance management in Italian manufacturing firms: Matters of size and matters of strategy. Journal of Quality in Maintenance Engineering, v.16, n.2, p.156-180, 2010. http://dx.doi.org/10.1108/13552511011048904

DURAN, O.; BATOCCHIO, A. Na direção da Manufatura Enxuta através da J4000 e o LEM. Produção Online, v.3, n.2, p.1-23, 2003. http://dx.doi.org/10.14488/1676-1901.v3i2.619 
FERREIRA, R.; FALANI, S.; JERONIMO, T. Aplicação de Modelo Multicritério para Manutenção Preditiva com Uso de Técnicas de Termografia Anais do XXX ENEGEP Encontro Nacional de Engenharia de Produção. São Carlos: ABEPRO, 2010.

FLÓRIO FILHO, O.; ARAÚJO, S.; FLÓRIO, A. Detecção automática do aquecimento em componentes de um sistema industrial no reconhecimento de imagens térmicas. Anais do XXVI ENEGEP - Encontro Nacional de Engenharia de Produção. Fortaleza: ABEPRO, 2006.

FRENCH, S., Action research for practising managers. Journal of Management Development, v.28, n.3, p.187-204, 2009. http://dx.doi.org/10.1108/02621710910939596

GARCIA, M.; SANZ-BOBI, M.; PICO, J. SIMAP: Intelligent System for Predictive Maintenance: Application to the health condition monitoring of a windturbine gearbox. Computers in Industry, v.57, n.6, p.552-568, 2006. http://dx.doi.org/10.1016/j.compind.2006.02.011

GARG, A; DESHMUKH, S. Maintenance management: literature review and directions. Journal of Quality in Maintenance Engineering, v.12, n.3, p.205-238, 2006. http://dx.doi.org/10.1108/13552510610685075

GRALL, A.; DIEULLE, L.; BÉRENGUER, C.; ROUSSIGNOL, M. Continuous-time predictivemaintenance scheduling for a deteriorating system. IEEE Transactions on Reliability, v.51, n.2, p.141-150, 2002. http://dx.doi.org/10.1109/TR.2002.1011518

HASHEMIAN, H.; BEAN, W. State-of-the-Art Predictive Maintenance Techniques. IEEE Transactions on Instrumentation and Measurement, v.60, n.10, p.3480-3492, 2011. http://dx.doi.org/10.1109/TIM.2009.2036347

KARDEC, A.; NASCIF, J.; BARONI, T. Gestão Estratégica e Técnicas Preditivas. Rio de Janeiro: QualityMark, 2002.

LIMA, J.; SANTOS, A.; SAMPAIO, R. Sistemas de Gestão da Manutenção - Uma Revisão Bibliográfica Visando Estabelecer Critérios para Avaliação de Maturidade. Anais do XXX ENEGEP - Encontro Nacional de Engenharia de Produção. São Carlos: ABEPRO, 2010.

MENDES, A. Manutenção Centrada em Confiabilidade: uma abordagem quantitativa. Dissertação (Mestrado em Engenharia de Produção) -- Programa de Pós-Graduação em Engenharia de Produção, Universidade Federal do Rio Grande do Sul - UFRGS, Porto Alegre, RS, 2011.

MIRSHAWKA, V. Manutenção Preditiva: caminho para zero defeitos. São Paulo: Makron McGraw-Hill, 1991.

NEPOMUCENO, L. Manutenção Preditiva em Instalações Industriais. São Paulo: Edgard Blücher, 1985.

NOGUEIRA, J.; REIS, V. Emprego da termografia na inspeção preditiva. Bolsista de Valor, v.1, n.1, p. 29-36, 2010.

OTANI, M.; MACHADO, W. A proposta de Desenvolvimento de Gestão da Manutenção Industrial na Busca da Excelência ou Classe Mundial. Gestão Industrial, v.4, n.2, p.1-16, 2008. http://dx.doi.org/10.3895/s1808-04482008000200001 
PEREIRA, M.; LIMA, W.; MONTEIRO, D. Sistema Computacional para Monitoramento OnLine de Vibração. Engenharia de Computação em Revista, v.1, n.4, p.1-6, 2010.

PERES, C.; LIMA, G. Proposta de modelo para controle de custos de manutenção com enfoque na aplicação de indicadores balanceados. Gestão \& Produção, v.15, n.1, p.149158, 2008. http://dx.doi.org/10.1590/S0104-530X2008000100013

RAMOS FILHO, J.; ATAMANCZUK, M.; MARÇAL, R. Seleção de técnicas de manutenção para processo de armazenagem pelo Método de Análise Hierárquica. Produção Online, v.10, n.1, p.142-166, 2010. http://dx.doi.org/10.14488/1676-1901.v10i1.375

SANTOS, G.; PEREIRA, J. Utilização da Análise de Componentes Principais em Termografia. Saber Acadêmico, v.10, n.2, p,15-24, 2010.

SCHIRMER, W.; LACEY, M.; LISBOA, H.; MIRANDA, G. Características, Natureza e Métodos de Amostragem/Análise de Gases Odorantes Emitidos em Processos Industriais: Caso das Lagoas de Tratamento de Efluentes. Revista de Ciências Ambientais, v.1, n.2, p.35-52, 2007.

SELLITTO, M. Inteligência artificial: uma aplicação em uma indústria de processo contínuo. Gestão \& Produção, v.9, n.3, p.363-376, 2002. http://dx.doi.org/10.1590/S0104530X2002000300010

SELLITTO, M. Formulação estratégica da manutenção industrial com base a confiabilidade dos equipamentos, Produção, v.15, n.1, p.44-59, 2005. http://dx.doi.org/10.1590/S0103-65132005000100005

SELLITTO, M.; BORCHARDT, M.; ARAÚJO, D. Manutenção centrada em confiabilidade: aplicando uma abordagem quantitativa. Anais do XXII ENEGEP, Encontro Nacional de Engenharia de Produção. Curitiba: ABEPRO, 2002.

SOLA, A.; XAVIER, A.; KOVALESKI, J.; RESENDE, L. Análise dos Fatores Determinantes para Eficiência Energética. Produção Online, v.6, n.1, p.1-26, 2008.

http://dx.doi.org/10.14488/1676-1901.v6i1.86

SOUZA, M. Métodos Analíticos para Lubrificantes e Isolantes. Química e Derivados, n.382, p.20-28, 2000.

SWANSON, L. An information-processing model of maintenance management. International Journal of Production Economics, v.83, n.1, p.45-64, 2003. http://dx.doi.org/10.1016/S0925-5273(02)00266-9

TACQUES, R.; SOUZA, M.; MATIAS, J.; GUILHERME, S. Vibração de Turbomáquinas: O System 1 na visão de usuário. Revista Abendi, v.6, n.31, p.22-29, 2009.

TARPANI, J.; ALMEIDA, E.; SIMÊNCIO, E.; MOTA, L., PAZ, J.; GUALBERTO, A.; CARDOSO, F.; GATTI. C. Inspeção termográfica de danos por impacto em laminados de matriz polimérica reforçados por fibras de carbono. Polímeros, v.19, n.4, p.318-328, 2009. http://dx.doi.org/10.1590/S0104-14282009000400012

TSANG, A. Condition based maintenance: tools and decision making. Journal of Quality in Maintenance Engineering, v.1, n.3, p.3-17, 1995.

http://dx.doi.org/10.1108/13552519510096350 
VIEIRA, A.; DALAVIA, F.; OLIMPIO, W.; SOLIS, T.; SANTOS, F.; ABADE, K. Controle de Contaminação para Fluídos Lubrificantes. ANAIS do XXVII ENEGEP- Encontro Nacional de Engenharia de Produção. Foz do Iguaçu: ABEPRO, 2007.

YA'CUBSOHN, R. El Diagnostico de fallas por análisis vibratorio. São Paulo: Die Techik Ltda., 1983.

YOU, M.; LI, L.; MENG, G.; NI, J. Cost-effective updated sequential predictive maintenance policy for continuously monitored degrading systems. IEEE Transactions on Automation Science and Engineering, v.7, n.2, p.257-265, 2010.

http://dx.doi.org/10.1109/TASE.2009.2019964

ZHOU, X.; XI, L.; LEE, J. Reliability-centered predictive maintenance scheduling for a continuously monitored system subject to degradation. Reliability Engineering \& System Safety, v.92, n.4, p.530-534, 2007. http://dx.doi.org/10.1016/j.ress.2006.01.006

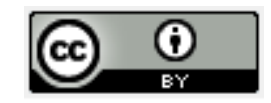

Artigo recebido em 15/06/2013 e aceito para publicação em 16/05/2014

DOI: http://dx.doi.org/ 10.14488/1676-1901.v15i3.1623 